\title{
Experimental and Computational Techniques in Soft Condensed Matter Physics
}

edited by Jeffrey S. Olafsen, Cambridge University Press, Cambridge, 2010, pp. ix +328 . Scope: monograph. ISBN 9780521115902 (hardback). Level: researchers and postgraduate physicists.

The "soft" part of condensed matter physics is distinctively different from the rest of the sub-discipline. The systems needing to be studied and understood are usually noncrystalline, and neither are they liquids or glasses in the conventional senses. In order to investigate and describe them, one needs to work in the interface regions between physics, chemistry, biology and engineering, drawing on ideas and techniques from all of these areas. Some understanding of nonlinear dynamics and complex systems is often needed too. The book that Jeffrey Olafsen has created is aimed at physicists and, in particular, at graduate students and experienced researchers entering the soft matter arena. As well as introducing them to the experimental and computational techniques that they will need, it is intended to supplement what physicists are usually taught about equilibrium thermodynamics and statistical mechanics. Readers should end up knowing enough about far-from-equilibrium phenomena and nonlinear dynamics to be able to embark on a range of topical problems related to the physics of soft matter.

The book consists of a collection of eleven mostly single-author review articles. It starts with Chapter 1 on the microscopy of soft materials. This is helpful, given that so much information and understanding of soft matter has been acquired by such means, and it links naturally to many of the later chapters. They range from computational methods for studying jammed systems, through Langmuir monolayers, granular systems, and cellular automata to structural patterns in bacterial colonies. Each chapter is self-contained, and opens with its own introduction and apologia for the topic to be discussed. We are reminded e.g. of the ubiquity of granular materials and that, arguably, particulate media are second only to water as the most manipulated material for human usage. Probably on account of the wide range of the topics being treated, there is not much repetition. There are some cross-references between the different chapters, and especially from Chapter 1 to the others. All of the chapters have extensive lists of references to the original literature and to reviews. 
The fact that there is neither a foreword nor a preface means that there is no clue as to the circumstances that led to the book being written. More seriously, at first sight, there is no introductory chapter to set the scene and draw the book together into an entity. Although I was initially disappointed by these lacunae, they probably do not matter very much. Most of the chapters are well-written, the material is interesting and important and, as already mentioned, there is some integration from the cross-referencing between chapters as well as from the themes themselves. I think that the book will be found useful.

\author{
Peter V. E. McClintock \\ Lancaster University \\ p.v.e.mcclintock@lancaster.ac.uk
}

